\title{
Self-similar sprays with heat and mass transfer
}

\author{
Hannes Hinterbichler, Helfried Steiner, Günter Brenn \\ Institute of Fluid Mechanics and Heat Transfer, Graz University of Technology, Graz, Austria \\ ${ }^{*}$ Corresponding author email: guenter.brenn@tugraz.at
}

\begin{abstract}
In pressure-atomized sprays, the liquid drops and the gas in the two-phase flow field may exhibit self-similar properties, so that the spray flow as a whole may be described as self-similar [1]. This description is used to model the mass transfer from the drops to the ambient gas by evaporation of the drop liquid. The concentration of the vapour phase is described as the solution of the related, well-known transport equation in a self-similar form. The self-similar transform of the mass source of vapour representing the effect of drop evaporation is compared against profiles obtained from the Steinberger and Treybal correlation for the Sherwood number as a function of the Schmidt and Reynolds numbers. The required surface concentration is based on the wet-bulb temperature of the drops, which was determined dependent on the local vapour content at ambient air temperature. The self-similar behavior of the vapour concentration and the respective mass source remains to be validated against experiments or simulations.
\end{abstract}

\section{Keywords}

pressure atomization, drop formation, self-similar flow field, drop evaporation, heat transfer

\section{Introduction}

Sprays are produced by atomization of a liquid in an ambient gaseous medium. In twin-fluid, air-assisted atomization, Li and Shen [2] and Ariyapadi et al. [3] found that the mean axial drop velocity may exhibit self-similar profiles. In [4-6] the spray was modelled as a self-similar single-phase jet with variable density, resulting in similar scaling variables as obtained for the single-phase case. In [7], Diesel-type sprays were investigated and self-similar axial velocity profiles were reported far downstream from the nozzle exit, where drops and gas phase are assumed to be in dynamic equilibrium. The authors of [8] found self-similar regions of normalized mean drop velocities and normalized Sauter-mean diameters in sprays from liquid-liquid coaxial swirl atomizers.

In pressure-atomization by ejection of a liquid into a gaseous environment, a flow field is induced in the otherwise quiescent gas phase. Recently, it has been shown that the progression of this type of spray flow towards an equilibrium state also exhibits self-similarity [1]. The self-similar analysis provided an analytical description of the gas flow field and of the rate of momentum transferred from the liquid to the gas phase.

We use the self-similar description of the spray flow [1], valid in the region dominated by momentum transfer from the droplets to the gas phase, to derive a self-similar model describing evaporating sprays. For this purpose we use the measurement data obtained by [1] with phaseDoppler anemometry (PDA). The PDA measurement data includes local drop characteristics at high spatial resolution, acquiring two velocity components and the size of $10^{5}$ drops at each point of measurement in the flow field. This method provides high statistical reliability of the drop data, even at the edges of the drop spectra. The sprays are generated by a single-phase pressure atomizer with off-axis liquid supply. The nozzle diameter is $0.4 \mathrm{~mm}$. The atomization outcome is characterized by the liquid Weber number in the nozzle orifice $W e_{l}$ and the liquid Ohnesorge number $\mathrm{Oh}_{l}$. Table 1 lists the values of these characteristic numbers together with the liquid mass flow rate $\dot{m}_{l}$ through the atomizer orifice. A detailed description of the experimental setup and the measurement results can be found in [1]. In the present study, the PDA measurement data is used to model the source of vapour in the sprays due to droplet evaporation. 
Table 1. Parameters of the experiments according to [1]. In the definitions of the liquid Weber and Ohnesorge numbers, $d_{o r}$ denotes the diameter of the atomizer orifice, $u_{o r}$ the mass-flow mean liquid velocity through the atomizer orifice, $\rho_{l}$ the liquid density, $\mu_{l}$ the liquid dynamic viscosity and $\sigma$ the liquid surface tension against air. For sprays 1 and 2, water is used as the test liquid. Spray 3 uses a mixture of ethanol and water with 10 mass percent ethanol.

\begin{tabular}{|c|c|c|c|}
\hline & Spray 1 & Spray 2 & Spray 3 \\
\hline$\dot{m}_{l}[\mathrm{~g} / \mathrm{s}]$ & 2.00 & 2.92 & 2.45 \\
\hline$W e_{l}=\rho_{l} d_{o r} u_{o r}^{2} / \sigma$ & 1395 & 2974 & 2921 \\
\hline$O h_{l}=\mu_{l} /\left(\rho_{l} \sigma d_{o r}\right)^{1 / 2}$ & 0.0059 & 0.0059 & 0.0099 \\
\hline
\end{tabular}

\section{Self-similar momentum transport}

Self-similar momentum transport in pressure-atomized spray flow, in the region of the sprays where momentum is transferred from the liquid to the gas phase, i.e. with large slip velocities between the drops and the gas, has recently been reported in [1]. The self-similar description of the gas flow field in the spray flow, accounting for momentum transfer between the phases, is reviewed in the present section and extended to account for self-similar heat and mass transfer in the next section.

The mathematical description of the gas flow is based on a boundary-layer approximation with negligible azimuthal velocity component and constant pressure throughout. The contribution of the viscous shear stress is neglected against the turbulent contribution, which is modeled using the Boussinesq eddy viscosity concept. Accordingly, the two-dimensional axisymmetric boundary-layer equations read

$$
\begin{aligned}
& \frac{\partial u_{z}}{\partial z}+\frac{1}{r} \frac{\partial\left(r u_{r}\right)}{\partial r}=0 \\
& \text { continuity } \\
& u_{z} \frac{\partial u_{z}}{\partial z}+u_{r} \frac{\partial u_{z}}{\partial r}=\nu_{t} \frac{1}{r} \frac{\partial}{\partial r}\left(r \frac{\partial u_{z}}{\partial r}\right)+f_{d, z} \\
& z \text {-momentum }
\end{aligned}
$$

The gas velocity components in axial and radial directions are denoted by $u_{z}$ and $u_{r}$, respectively. $\nu_{t}$ is the constant turbulent eddy viscosity and $f_{d, z}$ represents the momentum source due to momentum transfer from the liquid drops to the gas, which couples the axial gas momentum balance (2) to the axial momentum balance of the liquid phase [1]. The ansatz for the self-similar solution reads

$$
\eta=D \frac{r}{\left(z-z_{0}\right)^{\alpha}}, \quad \Psi=C\left(z-z_{0}\right) f(\eta), \quad f_{d, z}=C^{2} D^{4}\left(z-z_{0}\right)^{1-4 \alpha} \Omega_{z}(\eta) .
$$

Here, $\eta$ represents the self-similar coordinate and $\Psi$ the Stokesian stream function. $f(\eta)$ and $\Omega_{z}(\eta)$ denote the self-similar shape functions of the gas flow field and of the momentum source, respectively. $z_{0}$ is the virtual origin of the flow field. The exponent $\alpha$ determines the curvature of lines $\eta=$ constant in the $(r, z)$ space. The constants $C$ and $D$ are required for dimensional reasons. The values for the model constants reported by [1] are listed in table 2 . The exponent $\alpha$ is of approximately constant value, $\alpha=2 / 3$, in the three sprays.

The axial and radial velocity components calculated from the Stokesian stream function as per

$$
\begin{aligned}
& u_{z}=\frac{1}{r} \frac{\partial \Psi}{\partial r}=C D^{2}\left(z-z_{0}\right)^{1-2 \alpha} \frac{f^{\prime}}{\eta} \\
& u_{r}=-\frac{1}{r} \frac{\partial \Psi}{\partial z}=C D\left(z-z_{0}\right)^{-\alpha}\left(\alpha f^{\prime}-\frac{f}{\eta}\right)
\end{aligned}
$$

satisfy the continuity equation (1). Accordingly, we transform the $z$-momentum equation of the gas phase (2) into the self-similar representation

$$
(1-2 \alpha) f^{\prime 2}-\eta f\left(\frac{f^{\prime}}{\eta}\right)^{\prime}=\frac{\nu_{t}}{C} \eta\left[\eta\left(\frac{f^{\prime}}{\eta}\right)^{\prime}\right]^{\prime}+\eta^{2} \Omega_{z} .
$$


Table 2. Model parameters reported in [1]. $\nu=15.4 \mathrm{~mm}^{2} / \mathrm{s}$ denotes the kinematic viscosity of air at $20^{\circ} \mathrm{C}$.

\begin{tabular}{lccc}
\hline & Spray 1 & Spray 2 & Spray 3 \\
\hline$\alpha$ & 0.66 & 0.71 & 0.65 \\
$z_{0}[\mathrm{~mm}]$ & -2.2 & -14.8 & -3.1 \\
$C\left[\mathrm{~m}^{2} / \mathrm{s}\right]$ & 0.0033 & 0.0051 & 0.0070 \\
$D\left[\mathrm{~m}^{\alpha-1}\right]$ & 21.9 & 18.5 & 17.3 \\
$\nu_{t}\left[\mathrm{~mm}^{2} / \mathrm{s}\right]$ & $120 \nu$ & $150 \nu$ & $165 \nu$ \\
\hline
\end{tabular}

This equation is satisfied by the following solutions for the self-similar shape functions

$$
\begin{aligned}
& f(\eta)=\frac{\eta^{2}}{1+\eta^{2} / 4}, \\
& \Omega_{z}(\eta)=\frac{2}{\left(1+\eta^{2} / 4\right)^{4}}\left[2(1-2 \alpha)+\eta^{2}+\frac{\nu_{t}}{C}\left(2-\eta^{2}\right)\right] .
\end{aligned}
$$

Figure 1 (a) depicts the self-similar shape function of the scaled axial gas velocity $u_{z} / u_{z, 0}$, which can be determined from (4) and (7) as

$$
\frac{u_{z}}{u_{z, 0}}=\frac{f^{\prime}(\eta)}{\eta f^{\prime \prime}(0)}=\frac{1}{\left(1+\eta^{2} / 4\right)^{2}} \text {. }
$$

It is universal in the sprays investigated and bell-shaped with a peak at the symmetry axis. $u_{z, 0}$ denotes the axial gas velocity at the spray axis (at $\eta=0$ ). The self-similar shape functions of the momentum source $\Omega_{z}(\eta)$ are shown in figure $1(\mathrm{~b})$. They differ between the three sprays. In spray 1 , a bell-shaped curve with a maximum at the spray axis can be observed. In sprays 2 and 3 , the profiles exhibit a local minimum at the spray axis and an off-axis peak. The shapes of the profiles reflect the different regimes of atomization. In spray 1, at a lower Weber number, the conical sheet emerging from the orifice is not fully open, which corresponds to the so-called tulip-stage atomization regime. In the two other sprays, the sheet is open, which leads to finer atomization and a larger concentration of liquid mass and momentum at the location of the liquid sheet. This is indicated by the off-axis peaks in the profiles shown in figure 1(b).

The gas-phase momentum flow rate through every cross section $z=$ constant increases with the axial distance from the atomizer due to the momentum source in equation (2). Its axial scaling can by derived with the self-similar description of the gas flow field from the general definition of the axial gas momentum flow rate

$$
\mathcal{I}(z)=2 \pi \rho \int_{r=0}^{\infty} u_{z}^{2} r \mathrm{~d} r=2 \pi \rho C^{2} D^{2}\left(z-z_{0}\right)^{2-2 \alpha} \int_{\eta=0}^{\infty} \frac{f^{\prime 2}}{\eta} \mathrm{d} \eta=\frac{16}{3} \pi \rho C^{2} D^{2}\left(z-z_{0}\right)^{2-2 \alpha} .
$$

Here, $\rho$ represents the gas density. With values of $\alpha<1$, as listed in table 2 , the global gas momentum flow rate increases with $z$. Its axial evolution in the three sprays is depicted by figure 2(a). Similar to the global gas momentum flow rate, the gas mass flow rate, $\dot{m}$, can be derived from the self-similar description. It is defined as

$$
\dot{m}=2 \pi \rho \int_{r=0}^{\infty} u_{z} r \mathrm{~d} r=2 \pi \rho C\left(z-z_{0}\right) \int_{\eta=0}^{\infty} f^{\prime} \mathrm{d} \eta=8 \pi \rho C\left(z-z_{0}\right) .
$$

It is interesting to note that the exponent $\alpha$ does not appear in (11). This implies that the axial dependency of the mass flow rate, $\dot{m} \propto\left(z-z_{0}\right)$, does not change with the value in the exponent of the axial dependency of the gas momentum flow rate, $\mathcal{I} \propto\left(z-z_{0}\right)^{2-2 \alpha}$, for all types of flow described by equation (6). This observation is independent of the mathematical solution for the self-similar shape functions $f(\eta)$ and $\Omega_{z}(\eta)$. The axial evolution of the gas mass flow rate (11) is depicted in figure 2(b) for the three sprays. 

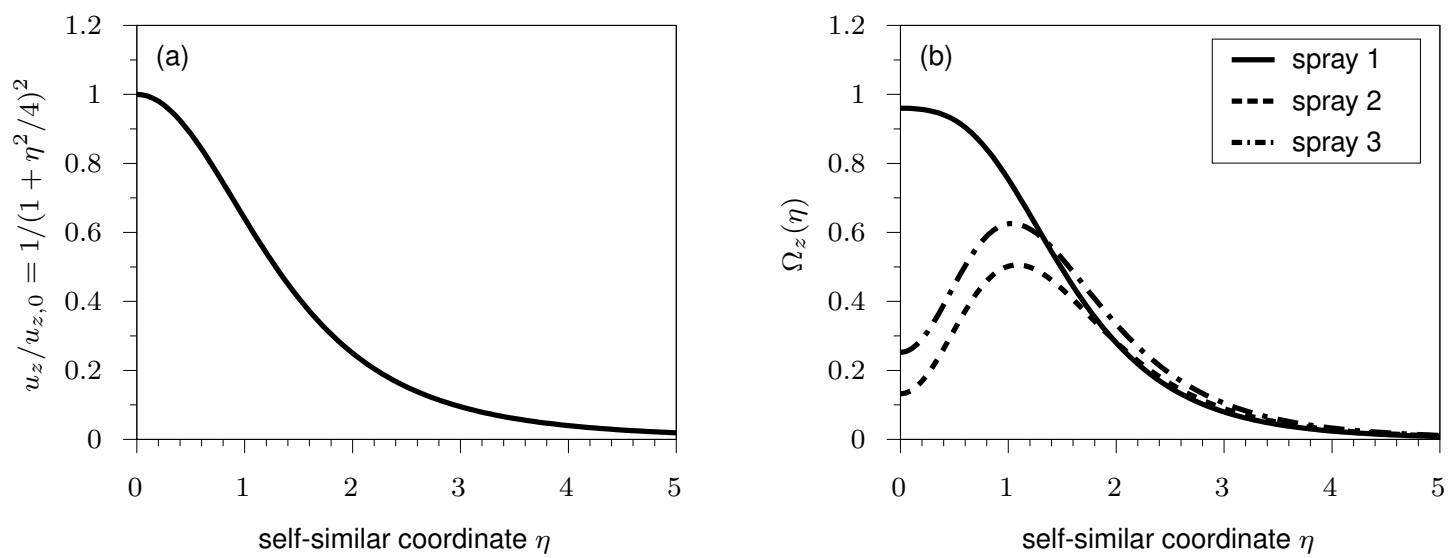

Figure 1. (a) Universal self-similar axial gas velocity profile (9). (b) Self-similar shape functions of the momentum source term (8) in the three sprays. The parameters of the self-similar description are listed in table 2.
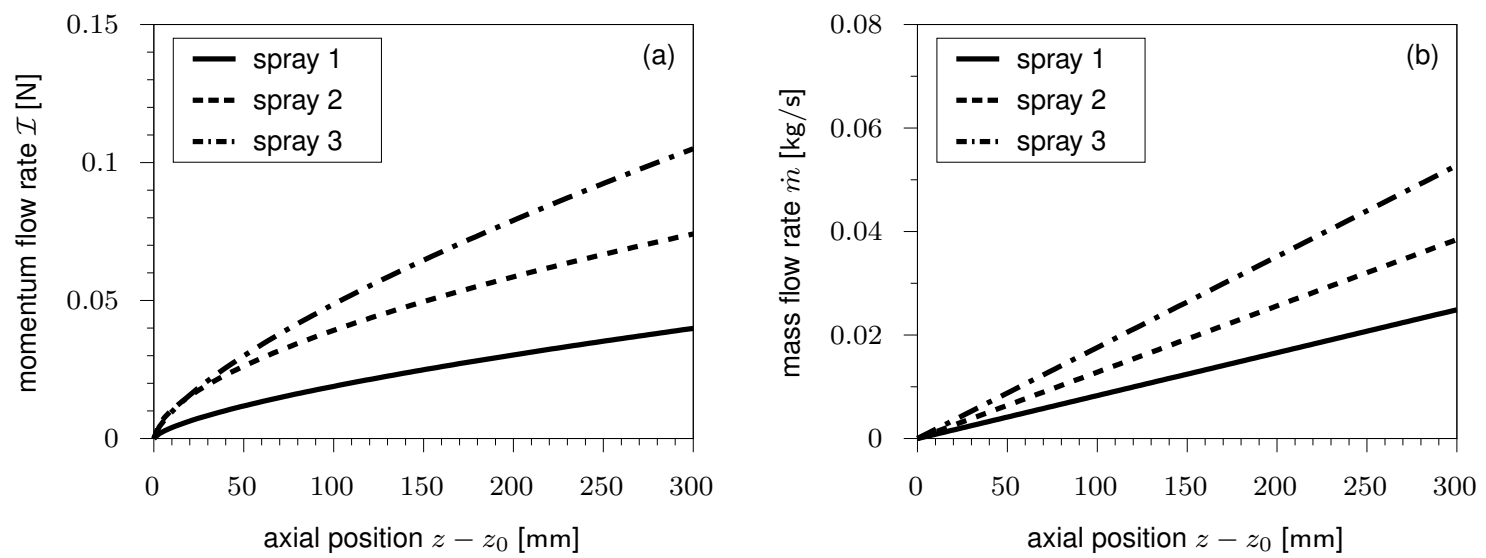

Figure 2. (a) Axial evolution of the gas momentum flow rate according to (10). (b) Linear increase of the gas mass flow rate calculated by (11). The parameters of the self-similar description for the three sprays are listed in table 2.

\section{Mass and heat transfer}

The self-similar transport equations for sprays with heat and mass transfer are again derived from boundary-layer theory.

\section{Self-similar mass transport}

The analysis of self-similar spray flow with account for momentum transfer, as reported in [1] and summarized in the previous section, is extended to describe mass transport in evaporating sprays. Based on the boundary-layer approximation, we describe the transport of the vapour component concentration $\rho_{i}$ in axisymmetric cylindrical form as

$$
u_{z} \frac{\partial \rho_{i}}{\partial z}+u_{r} \frac{\partial \rho_{i}}{\partial r}=\mathcal{D}_{t} \frac{1}{r} \frac{\partial}{\partial r}\left(r \frac{\partial \rho_{i}}{\partial r}\right)+S_{i}
$$

The effective turbulent diffusion coefficient $\mathcal{D}_{t}$ is assumed to be of constant value throughout the flow field. $S_{i}$ denotes the vapour source term due to evaporation of the liquid with density $\rho_{l, i}$. We base our analysis on the following assumptions. The presence of the vapour component $i$ does not influence the physical properties of the gas flow field, i.e. $\rho$ and $\nu$, and therefore does not change the velocity field determined by equations (1) and (2). Moreover, temperature changes due to latent heat are not considered.

We introduce an ansatz for the vapour concentration $\rho_{i}$ to transform the transport equation (12) 
into the self-similar coordinate. It reads

$$
\rho_{i}(r, z)-\rho_{i, \infty}=t(z) \Theta(\eta),
$$

where $\rho_{i, \infty}$ is the vapour concentration in the far field $(\eta \rightarrow \infty) \cdot t(z)$ denotes a scaling function and $\Theta(\eta)$ represents the self-similar vapour concentration. Introduction of (13) into (12) yields

$$
C\left(z-z_{0}\right) \frac{t^{\prime}}{t} f^{\prime} \Theta-C f \Theta^{\prime}=\mathcal{D}_{t}\left(\eta \Theta^{\prime}\right)^{\prime}+\frac{\left(z-z_{0}\right)^{2 \alpha}}{D^{2} t} \eta S_{i},
$$

where the prime denotes the derivative with respect to $z$ for $t(z)$ and with respect to $\eta$ for $f(\eta)$ and $\Theta(\eta)$. For (14) to become an ordinary differential equation, independent of $z$, we require

$$
C\left(z-z_{0}\right) \frac{t^{\prime}}{t}=\text { constant }:=E
$$

resulting in

$$
t(z)=F\left(z-z_{0}\right)^{E / C}
$$

with the two, yet unknown, constants $E$ and $F$. Moreover, for self-similarity, we require the vapour source term $S_{i}$ to be of the form

$$
S_{i}(r, z)=\mathcal{D}_{t} D^{2}\left(z-z_{0}\right)^{-2 \alpha} t(z) \Phi_{i}(\eta),
$$

where $\Phi_{i}(\eta)$ denotes the yet unknwon self-similar shape function of the vapour source. For convenience, we introduce the constant

$$
\beta:=\frac{E}{C} \text {. }
$$

Thus, the ansatz for the vapour concentration (13) and the vapour source (17) turn into

$$
\rho_{i}(r, z)-\rho_{i, \infty}=F\left(z-z_{0}\right)^{\beta} \Theta(\eta), \quad S_{i}(r, z)=\mathcal{D}_{t} D^{2} F\left(z-z_{0}\right)^{\beta-2 \alpha} \Phi_{i}(\eta) .
$$

The constant $F$ is required for dimensional reasons. The exponent $\beta$ determines the axial scaling of the vapour concentration and the vapour source. Introducing the turbulent Schmidt number $S c_{t}=\nu_{t} / \mathcal{D}_{t}$, the self-similar transport equation (14) becomes

$$
S c_{t} \frac{C}{\nu_{t}}\left(\beta f^{\prime} \Theta-f \Theta^{\prime}\right)=\left(\eta \Theta^{\prime}\right)^{\prime}+\eta \Phi_{i} .
$$

With $\beta=-1$ and $\Phi_{i}=0$, (20) turns into the well-known transport equation of the single-phase round jet [9]. The solution of equation (20) is subject to two boundary conditions. We define them by considering the symmetry of the problem and the vapour concentration in the far field

$$
\begin{array}{rll}
\left.\frac{\partial \rho_{i}}{\partial \eta}\right|_{\eta \rightarrow 0}:=0 & \Rightarrow & \Theta^{\prime}(0)=0, \\
\left.\rho_{i}\right|_{\eta \rightarrow \infty}:=\rho_{i, \infty} & \Rightarrow & \Theta(\eta \rightarrow \infty)=0 .
\end{array}
$$

Knowledge of the exponent $\beta$, the constant $F$, the turbulent Schmidt number $S c_{t}$ and the selfsimilar shape function of the vapour source $\Phi_{i}$ is required to determine the self-similar vapour concentration profile $\Theta(\eta)$ from (20). Concerning the turbulent Schmidt number, Tominaga and Stathopoulos [10] suggest a value of $S c_{t}=0.9$ in turbulent single-phase free shear flow. The exponent $\beta$ relates to the global mass flow rate of the vapour component concentration $\rho_{i}$ as per

$$
\dot{m}_{i}=2 \pi \int_{r=0}^{\infty} u\left(\rho_{i}-\rho_{i, \infty}\right) r \mathrm{~d} r=2 \pi C F\left(z-z_{0}\right)^{1+\beta} \int_{\eta=0}^{\infty} f^{\prime} \Theta \mathrm{d} \eta
$$



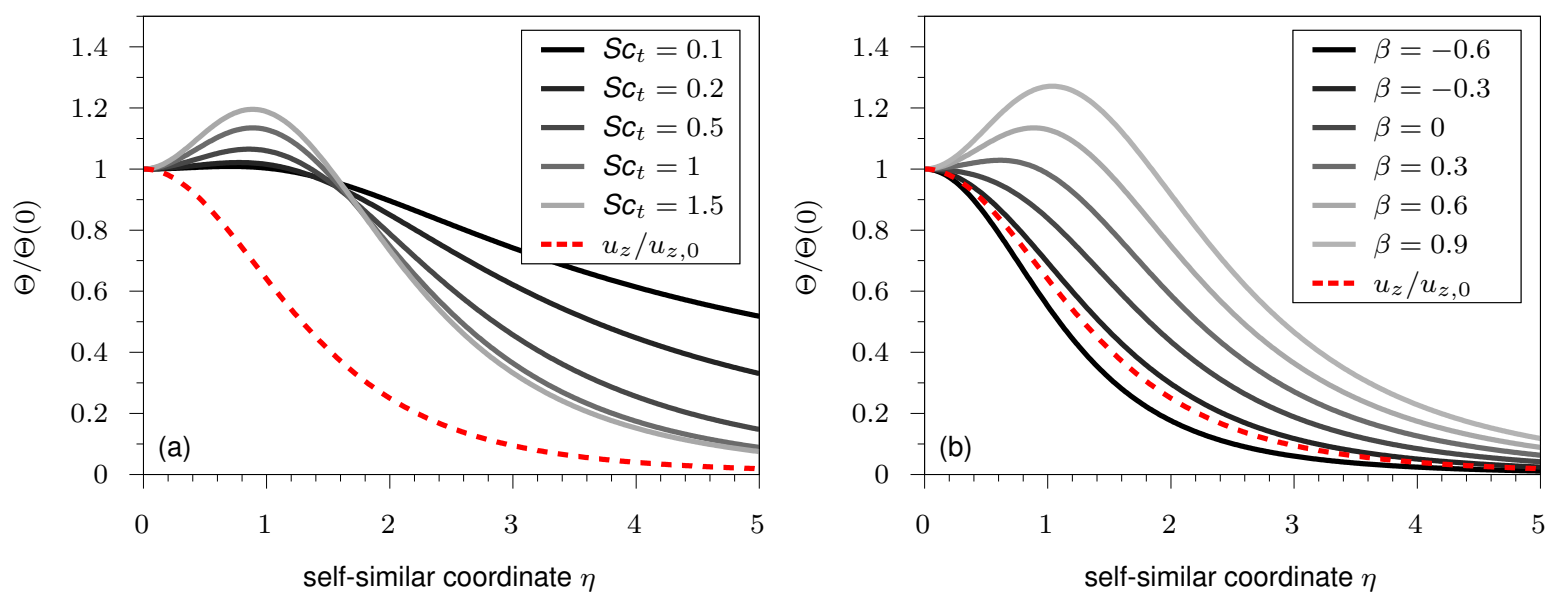

Figure 3. Self-similar vapour concentration profiles obtained from (20) for (a) $\beta=0.6$ and (b) $S c_{t}=1$ in spray 2 .

In the case of an increasing mass flow rate, i.e. evaporation of the liquid with density $\rho_{l, i}$, we expect a value of $\beta>-1$. To determine the exact values of the model parameters, measurement data on the vapour concentration field in such spray flows is required. With information on the local concentration field $\rho_{i}(r, z)$, self-similar behavior could be verified and the parameters $\beta$ and $F$, as well as the self-similar concentration profile $\Theta(\eta)$ could be determined. Subsequently, from (20) the shape of the vapour source $\Phi_{i}(\eta)$ could be determined and compared to modeling or simulation results. With the PDA measurement data at hand, this is beyond the scope of the present work and subject to future studies.

As an example, we determine possible self-similar concentration profiles from (20) for different values of the relevant parameters. For this purpose, we assume the self-similar shape function of the vapour source, $\Phi_{i}(\eta)$, to be equal to the self-similar shape function of the momentum source (8), i.e. $\Phi_{i}(\eta):=\Omega_{z}(\eta)$. In figure 3, hypothetical self-similar concentration profiles are depicted for various values of (a) $S c_{t}$ and (b) $\beta$. We used the self-similar parameters of spray 2 to obtain these profiles (see table 2). As expected, a smaller turbulent Schmidt number produces spatially more uniform vapour concentration profiles, reflecting the enhanced turbulent mixing of the vapour phase. Larger values of $\beta$ reflect a stronger increase in the vapour mass flow rate (23), resulting in wider self-similar concentration profiles and vice versa. For total analogy between mass and momentum transfer $\left(\Phi_{i}(\eta)=\Omega_{z}(\eta), \beta=1-2 \alpha, S c_{t}=1\right)$, the obtained concentration profile collapses with the self-similar axial gas velocity profile (dashed red line).

\section{Analytically based parameterisation of the vapour source model}

Due to the lack of experimental calibration data, or data from high-quality simulations, we propose an analytically based approach, which provides a relationship between the parameters $\beta$ and $F$, so that only one them remains to be specified. The proposed concept models the vapour source $S_{i}$ as being generated by the evaporation of an ensemble of single droplets using the well-known Sherwood-correlation of Frössling [11] for the mass transfer coefficient, which is written as

$$
S_{i}=\pi \mathcal{D} \sum_{j=1}^{J} d_{p, j} n_{j} \underbrace{\left(2+0.553 R e_{p, j}^{1 / 2} S c^{1 / 3}\right)}_{S h}\left(\rho_{i, s, j}-\rho_{i}\right) .
$$

Here, $d_{p, j}$ denotes the mean drop diameter in the $j$ th drop size class, $n_{j}$ the corresponding local drop-number concentration obtained from the PDA measurements, $R e_{p, j}$ the corresponding drop Reynolds number, and $J$ the total number of drop size classes. $S c$ is the Schmidt number, and $\rho_{i, s, j}$ the vapour concentration at the drop surface. The correlation (24) is evaluated along 

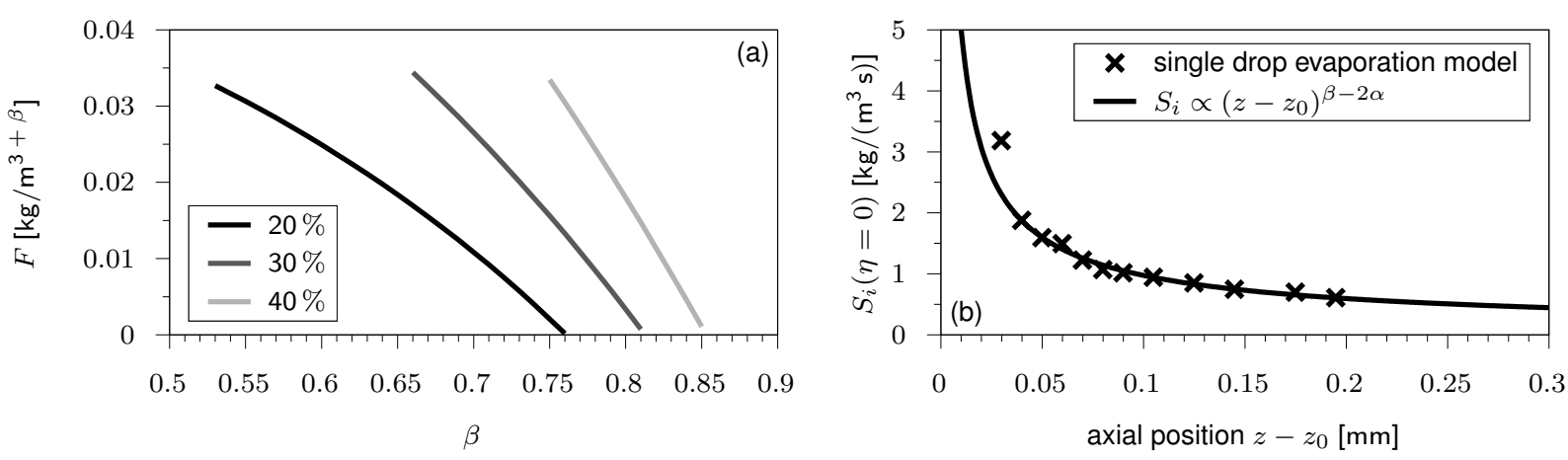

Figure 4. Spray 2: (a) Parameter $F$ vs. exponent $\beta$ for varying relative humidity of the far field, (b) predicted source term for $\beta=0.7, F(\beta)=0.0265 \mathrm{~kg} / \mathrm{m}^{3+\beta}$ at $30 \%$ relative humidity of the far field.

the axis of the spray supplied with the axial variation for the drop Reynolds number and the vapour concentration at the drop surface obtained as

$$
\boldsymbol{R e}_{p}=\frac{d_{p}\left|u_{p}-u\right|}{\nu}, \rho_{i, s}=\rho\left[1+\left(\frac{p}{\exp \left(77.345+0.0057 T_{p}-7235 / T_{p}\right) / T_{p}^{8.2}}-1\right) \frac{M_{g}}{M_{i}}\right]^{-1},
$$

respectively. The axial variations of the droplet diameter $d_{p}$, velocity $u_{p}$ and temperature $T_{p}$ required in (25) are analytically obtained by integrating balances of drop mass, momentum and energy

$$
\begin{aligned}
\frac{\mathrm{d} d_{p}}{\mathrm{~d} z} & =\frac{2 \nu}{u_{p} \rho_{p} d_{p}} \frac{S h}{S c}\left(\rho_{i}-\rho_{i, s}\right) \\
\frac{\mathrm{d} u_{p}}{\mathrm{~d} z} & =\frac{18 \nu \rho}{u_{p} \rho_{p} d_{p}^{2}}\left(1+\frac{1}{6} R e_{p}^{0.8}\right)\left(u-u_{p}\right) \\
\frac{\mathrm{d} T_{p}}{\mathrm{~d} z} & =\frac{6 \nu}{u_{p} d_{p}^{2} S c} \frac{\rho}{\rho_{p}} \frac{c}{c_{p}} S h\left[\frac{h_{l g}}{\rho c}\left(\rho_{i}-\rho_{i, s}\right)+\operatorname{Le}\left(T_{\infty}-T_{p}\right)\right],
\end{aligned}
$$

respectively, along the spray axis $z$, which essentially follows a concept of Abramzon and Sirignano [12]. The self-similar solution of the momentum equation (4) is used for the velocity of the gas phase, $u=u_{z}$, and the self-similarity ansatz (19) is substituted for the vapor concentration $\rho_{i}$. Appying a best fit of the correlation for $S_{i}(24)$ with the corresponding selfsimilar expression (19) over a given range of axial positions $z$ finally provides the sought relationship between the parameters $\beta$ and $F$. Figure 4(a) exemplarily demonstrates this relationship obtained for spray 2 , assuming $20 / 30 / 40 \%$ relative humidity in the far field. Figure 4(b) depicts the corresponding axial variation of the source term, as predicted by the selfsimilarity ansatz (19) and the correlation (24) for the particular choice $\beta=0.7$ at the relative humidity of $30 \%$, where $F(\beta)$ becomes $0.0265 \mathrm{~kg} / \mathrm{m}^{3}+\beta$. The good agreement, which can be evidently achieved with both descriptions for $S_{i}$, supports the reliability of the present method for coupling the parameters $\beta$ and $F$. Experimental data or data from simulations are still needed to complete the parametrization by determining either $\beta$ or $F$.

\section{Conclusions}

Based on the self-similar description of spray flow by [1], with strong momentum transfer from the liquid to the gas phase, we model the mass transfer from the drops to the gas phase by evaporation and derive a self-similar description of the transport equation for the concentration of the vapour phase from boundary-layer theory. Dependent on the model parameters, possible solutions for the self-similar concentration profiles are presented and discussed. Experimental data of the vapour concentration in sprays, as investigated, or data from high-quality simulations 
is required to determine the exact values of the model parameters. Future work will involve the determination of these parameters.

\section{Acknowledgments}

The authors gratefully acknowledge the financial support from the Austrian Research Promotion Agency FFG (project no. 849876). This work is supported by SIINN ERA-NET, funded under the ERA-NET scheme of the Seventh Framework Programme of the European Commission, Research Directorate - General, Grant Agreement No. 265799.

\section{Nomenclature}

$\begin{array}{ll}\text { Latin symbols } \\ a_{t} & \text { thermal diffusivity }\left[\mathrm{m}^{2} / \mathrm{s}\right] \\ c_{p} & \text { specific isobaric heat } \\ & \text { capacity }[\mathrm{J} /(\mathrm{kg} \mathrm{K})] \\ C & \text { model constant }\left[\mathrm{m}^{2} / \mathrm{s}\right] \\ d & \text { diameter [m] } \\ D & \text { model constant }\left[\mathrm{m}^{\alpha-1}\right] \\ \mathcal{D} & \text { diffusion coefficient }\left[\mathrm{m}^{2} / \mathrm{s}\right] \\ E & \text { constant }\left[\mathrm{m}^{2} / \mathrm{s}\right] \\ F & \text { model constant }\left[\mathrm{kg} / \mathrm{m}^{3}+\beta\right] \\ f(\eta) & \text { self-similar shape function } \\ f_{d} & \text { momentum source }\left[\mathrm{m}^{2} / \mathrm{s}^{2}\right] \\ \mathcal{H} & \text { enthalpy flow rate }[\mathrm{J} / \mathrm{s}] \\ \mathcal{I} & \text { momentum flow rate }[\mathrm{N}] \\ J & \text { number of drop } \mathrm{size} \mathrm{classes} \\ M & \text { molar mass }[\mathrm{kg} / \mathrm{mol}] \\ \dot{m} & \text { mass flow rate }[\mathrm{kg} / \mathrm{s}] \\ n & \text { drop-number concentration }\left[1 / \mathrm{m}^{3}\right] \\ p & \text { pressure }[\mathrm{Pa}] \\ r & \text { radial coordinate }[\mathrm{m}] \\ S_{i} & \text { vapour source }\left[\mathrm{kg} /\left(\mathrm{m}^{3} \mathrm{~s}\right)\right] \\ \mathcal{S}_{h} & \text { heat source }[\mathrm{K} / \mathrm{s}] \\ t(z) & \text { scaling function }\left[\mathrm{kg} / \mathrm{m}^{3}\right] \\ T & \text { temperature }[\mathrm{K}] \\ u & \text { velocity component }[\mathrm{m} / \mathrm{s}] \\ u_{0} & \text { velocity at the } \mathrm{spray} \mathrm{axis}[\mathrm{m} / \mathrm{s}] \\ z & \text { axial coordinate }[\mathrm{m}] \\ z_{0} & \text { virtual origin }[\mathrm{m}]\end{array}$

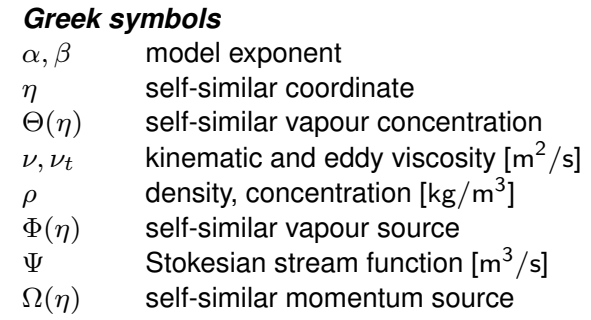

\section{Subscripts}

vapour component drop size class liquid phase liquid droplet quantity in radial direction at the drop surface turbulent in axial direction in the far field

$\begin{array}{ll}\text { Nondimensional groups } \\ \text { Le } & \text { Lewis number } \\ \mathrm{Oh} & \text { Ohnesorge number } \\ \mathrm{Pr} & \text { Prandtl number } \\ \mathrm{Re} & \text { Reynolds number } \\ \mathrm{Sc} & \text { Schmidt number } \\ \text { We } & \text { Weber number }\end{array}$

\section{References}

[1] Hinterbichler, H., Steiner, H., Brenn, G., 2020, Journal of Fluid Mechanics, 889, A17.

[2] Li, X., and Shen J., 1999, Journal of Propulsion and Power, 15, pp. 103-110.

[3] Ariyapadi, S., Balachandar, R., Berruti, F., 2003, The Canadian Journal of Chemical Engineering, 81, pp. 923-939.

[4] Faeth G. M., 1983, Progress in Energy and Combustion Science, 9, pp. 1-76.

[5] Panchagnula, M. V., and Sojka P. E., 1999, Fuel, 78, pp. 729-741.

[6] Desantes, J.M., Salvador, F.J., Lopez, J.J., De La Morena, J., 2011, Experiments in Fluids, 50, pp. 233-246.

[7] Wu, K.-J., Santavicca, D.A., Bracco, F.V., Coghe, A., 1984, AlAA Journal, 22, pp. 1236-1270.

[8] Soltani, M.R., Ghorbanian, K., Ashjaee, M., Morad, M.R., 2005, Aerospace Science and Technology, 9, pp. 592-604.

[9] Tropea, C., Yarin, A., Foss, J.F., 2007, Springer Handbook of Experimental Fluid Mechanics. Springer, Berlin Heidelberg.

[10] Tominaga, Y., and Stathopoulos, T., 2007, Atmospheric Environment, 41, pp. 8091-8099.

[11] Frössling, N., 1938, Gerlands Beiträge zur Geophysik, 52, pp. 170-216.

[12] Abramzon, B. and Sirignano, W.A., 1989, Int. J. Heat Mass Transfer, 32, pp. 1605-1618. 